On a limiting absorption principle for sesquilinear forms with an application to the Helmholtz equation in a waveguide

\author{
Ben Schweizer, Maik Urban
}




\title{
On a limiting absorption principle for sesquilinear forms with an application to the Helmholtz equation in a waveguide
}

\author{
Ben Schweizer, Maik Urban
}

\begin{abstract}
We prove a limiting absorption principle for sesquilinear forms on Hilbert spaces and apply the abstract result to a Helmholtz equation with radiation condition. The limiting absorption principle is based on a Fredholm alternative. It is applied to Helmholtz-type equations in a truncated waveguide geometry. We analyse a problem with radiation conditions on truncated domains, recently introduced in [4]. We improve the previous results by treating the limit $\delta \rightarrow 0$.
\end{abstract}

\section{Introduction}

This article is devoted to the analysis of time-harmonic versions of wave equations, the most prominent example being the Helmholtz equation $\mathcal{L} u:=-\nabla \cdot(a \nabla u)-\omega^{2} u=$ $f$, where $\omega$ is the prescribed frequency of the problem, $a$ a positive coefficient, and $f$ a source term. The equation is posed in a domain $\Omega \subset \mathbb{R}^{n}$ and it is complemented by a boundary condition on $\partial \Omega$. For applications, it is interesting to solve the equation in an unbounded domain $\Omega$. In order to make the equation well-posed in an unbounded domain, radiation conditions must be imposed in those directions in which $\Omega$ is unbounded.

A vast body of literature is devoted to radiation conditions such as the classical Sommerfeld condition and their analysis, we mention $[2,12,14]$ and the overview of [13]. Recently, the interest turned to radiation conditions in periodic media. This requires new methods, see $[5,6,10]$. Related to radiation conditions is the question of a numerical treatment of the equation; the domain must be truncated (replaced by a bounded domain), and the radiation condition must be replaced appropriately. Methods like non-reflecting boundary conditions or perfectly matched layers are

Ben Schweizer e-mail: ben.schweizer@tu-dortmund.de Technische Universität Dortmund, Fakultät für Mathematik, Vogelpothsweg 87, D-44227

Maik Urban e-mail: maik.urban@tu-dortmund.de Technische Universität Dortmund, Fakultät für Mathematik, Vogelpothsweg 87, D-44227 
successfully applied to problems with constant coefficients, but these methods cannot be used in a periodic medium. The approach of [4] is to introduce radiation boxes and to demand that the solution of the truncated problem is an outgoing wave in the radiation boxes. The problem can be formulated with the help of a sesquilinear form.

Limiting absorption principle. A tool to establish the existence of solutions to $\mathcal{L} u=f$ in an unbounded domain is the limiting absorption principle. The idea of that approach is to add a small absorption term. For $\delta>0$ we study

$$
\mathcal{L} u_{\delta}+\delta \mathrm{i} u_{\delta}=f,
$$

such that the original problem is recovered for $\delta=0$. Usually, it is easy to establish the existence of a solution to (1). Given a family of solutions $\left(u_{\delta}\right)_{\delta}$, we can study the limit $\delta \rightarrow 0$. Any limit of the sequence $\left(u_{\delta}\right)_{\delta}$ is a candidate for a solution to the original problem. This program has been performed successfully in several settings, as recent examples we mention [7] and [11].

A radiation condition for truncated domains was introduced in [4], the article contains a well-posedness result for positive absorption $\delta>0$. Here, we study the limit $\delta \rightarrow 0$ of that system.

Abstract setting. In the first part of this article, we derive the limiting absorption principle in an abstract setting, and consider sesquilinear forms on Hilbert spaces. We recall that the system of [4] is formulated with sesquilinear forms, hence our abstract results can be applied to this "radiation condition on truncated domains".

Given a (real or complex) Hilbert space $\mathcal{H}$, a sesquilinear form $b$, and $\ell \in \mathcal{H}^{*}$, we look for an element $u \in \mathcal{H}$ that satisfies the equation

$$
b(u, \cdot)=\ell(\cdot) .
$$

If $b$ is bounded and coercive, then the existence of such an element $u$ follows from the Lax-Milgram lemma. But how can we establish the existence of a solution $u \in \mathcal{H}$ to (2) if $b$ is not coercive? We perform the following approach: instead of $b$, we consider a family $\left(b_{\delta}\right)_{\delta}$ of sesquilinear forms such that there is a unique solution $u_{\delta} \in \mathcal{H}$ to

$$
b_{\delta}\left(u_{\delta}, \cdot\right)=\ell(\cdot)
$$

for each $\delta>0$. Under suitable assumptions on the family $\left(b_{\delta}\right)_{\delta}$, we prove that the sequence of solutions $\left(u_{\delta}\right)_{\delta}$ converges to a solution $u$ to (2) as $\delta \rightarrow 0$.

The limiting absorption principle is based on a Fredholm alternative for sesquilinear forms; see Proposition 2.1. We note that the Fredholm alternative is not new, but rather a variant of known results, see [8,9].

Application to the Helmholtz equation in periodic waveguide. Let us sketch the setting of the truncated problem of [4]. The geometry is described by the numbers $\varepsilon, R>0$ and $H \in \mathbb{N}$, the truncated domain is $\Omega_{R}:=(-\varepsilon R, \varepsilon R) \times(0, \varepsilon H)$. A parameter $L>0$ defines the width of the two radiation boxes $(\varepsilon R, \varepsilon(R+L)) \times(0, \varepsilon H)$ and $(-\varepsilon(R+L),-\varepsilon R) \times(0, \varepsilon H)$. The coefficient $a: \mathbb{R}^{2} \rightarrow \mathbb{R}$ is assumed to be periodic in the left half-space and in the right half-space; that is, $a(x)=a_{-}(x)$ for $x \in(-\infty, 0) \times(0, \varepsilon H)$ and $a(x)=a_{+}(x)$ for $x \in[0, \infty) \times(0, \varepsilon H)$, where $a_{-}$and $a_{+}$ 
are $\varepsilon$-periodic functions on $\mathbb{R} \times(0, \varepsilon H)$. The problem is to find a function $u_{\delta}$, which solves (1) in $\Omega_{R}$ and which is radiating in radiation boxes. This problem is recast in [4] with a sesquilinear form $\beta_{\delta}$ and is written as $\beta_{\delta}\left(u_{\delta}, \cdot\right)=\langle f, \cdot\rangle$.

The problem for $\delta>0$ was analysed in [4]. Our abstract results allow to study the limit $\delta \rightarrow 0$ : Except for a countable set of singular frequencies $\omega$, the solutions $u_{\delta}$ converge to the unique solution $u$ of the original problem $\beta(u, \cdot)=\langle f, \cdot\rangle$.

\section{Fredholm alternative and limiting absorption principle}

Our aim in this section is to present a limiting absorption principle for a sesquilinear form defined on a Hilbert space over $\mathbb{R}$ or $\mathbb{C}$. The limiting absorption principle is based on a Fredholm alternative, which we present first.

\subsection{Fredholm alternative for sesquilinear forms}

The Fredholm alternative for Fredholm operators of index 0 is a classical result in functional analysis. As we focus on problems involving sesquilinear forms rather than operators, we present a Fredholm alternative for sesquilinear forms.

We recall that such an alternative is new. Indeed, Kress [9] proved a Fredholm alternative for bilinear forms on reflexive Banach spaces and characterised the bilinear forms for which such a Fredholm alternative can hold. As our version of the Fredholm alternative is slightly different from the one presented by Kress and for convenience of the reader, we provide a proof.

Let $\mathcal{H}$ be a Hilbert space over the field $\mathbb{K}=\mathbb{R}$ or $\mathbb{K}=\mathbb{C}$ and let $b: \mathcal{H} \times \mathcal{H} \rightarrow \mathbb{K}$ be a sesquilinear form; that is, $b$ is anti-linear in its first argument and linear in the second. Note that $b$ is bilinear in case $\mathbb{K}=\mathbb{R}$. The norm on $\mathcal{H}$ is denoted by $\|\cdot\|$. The kernel of $b$ is defined as

$$
\operatorname{ker}(b):=\{u \in \mathcal{H}: b(u, v)=0 \text { for all } v \in \mathcal{H}\} .
$$

The adjoint $b^{*}: \mathcal{H} \times \mathcal{H} \rightarrow \mathbb{K}$ of $b$ is given by $b^{*}(u, v):=\overline{b(v, u)}$. In case $\mathbb{K}=\mathbb{R}$ this reduces to $b^{*}(u, v):=b(v, u)$. We say that a sesquilinear form $b: \mathcal{H} \times \mathcal{H} \rightarrow \mathbb{K}$ satisfies a Gårding inequality if there are a constant $c>0$ and a compact operator $K: \mathcal{H} \rightarrow \mathcal{H}^{*}$ such that

$$
\left|b(u, u)+\langle K u, u\rangle_{\mathcal{H}^{*}, \mathcal{H}}\right| \geq c\|u\|^{2},
$$

for all $u \in \mathcal{H}$. The space of bounded linear functionals is denoted by $\mathcal{H}^{*}$. Thus, for $\mathbb{K}=\mathbb{C}$ the compact operator $K: \mathcal{H} \rightarrow \mathcal{H}^{*}$ in the Gårding inequality is an anti-linear map. 
Proposition 2.1 (Fredholm alternative for sesquilinear forms) Let $\mathcal{H}$ be a Hilbert space, and let $b: \mathcal{H} \times \mathcal{H} \rightarrow \mathbb{K}$ be a bounded sesquilinear form that satisfies a Gårding inequality. Then the following statements hold:

(a) Let $\ell \in \mathcal{H}^{*}$. There exists $u \in \mathcal{H}$ with

$$
b(u, \cdot)=\ell(\cdot)
$$

if and only if

$$
\ell(v)=0 \quad \text { for all } v \in \operatorname{ker}\left(b^{*}\right) .
$$

(b) The dimension of $\operatorname{ker}(b)$ is finite and equals the dimension of $\operatorname{ker}\left(b^{*}\right)$.

(c) If $\operatorname{ker}(b)=\{0\}$, then for every $\ell \in \mathcal{H}^{*}$ there is a unique $u \in \mathcal{H}$ such that (5) holds.

Let us recall that a bounded linear operator $F: \mathcal{H} \rightarrow \mathcal{K}$ between two Hilbert spaces $\mathcal{H}$ and $\mathcal{K}$ is called a Fredholm operator if both its kernel $\operatorname{ker} F$ and its cokernel coker $F:=\mathcal{K} / \operatorname{im} F$ are finite dimensional. The index of $F$ is defined as

$$
\text { ind } F:=\operatorname{dim} \operatorname{ker} F-\operatorname{dim} \operatorname{coker} F \in \mathbb{Z} \text {. }
$$

The Fredholm index is stable under compact perturbations; that is, if $F$ is a Fredholm operator and $K: \mathcal{H} \rightarrow \mathcal{K}$ is compact, then $F+K$ is a Fredholm operator with $\operatorname{ind}(F+K)=\operatorname{ind} F$. Denoting the adjoint of $F$ by $F^{*}: \mathcal{K} \rightarrow \mathcal{H}$, one readily checks that $\operatorname{ker} F^{*} \cong \operatorname{coker} F$ and coker $F^{*} \cong \operatorname{ker} F$; in particular, $F^{*}$ is a Fredholm operator if and only if $F$ is, and we have ind $F=-$ ind $F^{*}$. For a discussion of Fredholm operators we refer to [3].

We call an anti-linear operator $F: \mathcal{H} \rightarrow \mathcal{K}$ an anti-linear Fredholm operator if $\mathcal{H} \rightarrow \mathcal{K}, u \mapsto \overline{F u}$ is a Fredholm operator. The following result will be useful in the proof of Proposition 2.1.

Lemma 2.1 Let $\mathcal{H}$ be a Hilbert space and let $b: \mathcal{H} \times \mathcal{H} \rightarrow \mathbb{K}$ be a bounded sesquilinear form that satisfies a Gårding inequality. Then the operator $B: \mathcal{H} \rightarrow \mathcal{H}^{*}$ given by $u \mapsto b(u, \cdot)$ is an anti-linear Fredholm operator of index 0 .

Proof The sesquilinear form $b$ is anti-linear an in its first argument and bounded, which implies anti-linearity and boundedness of $B$. By assumption, $b$ satisfies a Gårding inequality; hence there is a compact anti-linear operator $K: \mathcal{H} \rightarrow \mathcal{H}^{*}$ such that (4) holds. The operator $(B+K): \mathcal{H} \rightarrow \mathcal{H}^{*}$ induces a sesquilinear form $a: \mathcal{H} \times \mathcal{H} \rightarrow \mathbb{K}$

$$
a(u, v):=\langle(B+K) u, v\rangle_{\mathcal{H}^{*}, \mathcal{H}}=b(u, v)+\langle K u, v\rangle_{\mathcal{H}^{*}, \mathcal{H}} .
$$

Thanks to the boundedness of $b$ and $K$, the sesquilinear form $a$ is bounded as well. Moreover, $a$ is coercive since $b$ satisfies a Gårding inequality. We can apply the Lax-Milgram lemma to conclude that the operator $(B+K): \mathcal{H} \rightarrow \mathcal{H}^{*}$ is bijective. Consequently, $B+K$ is an anti-linear Fredholm operator of index 0 . As the Fredholm index is stable under compact perturbations, we deduce that $B=(B+K)-K$ is also an anti-linear Fredholm operator of index 0 . This proves the claim. 
Having Lemma 2.1 at hand, we can perform the proof of Proposition 2.1.

Proof (of Proposition 2.1) By Lemma 2.1 we know that the operator $B: \mathcal{H} \rightarrow \mathcal{H}^{*}$, $u \mapsto b(u, \cdot)$ is an anti-linear Fredholm operator of index 0. Obviously, $\operatorname{ker}(b)=\operatorname{ker} B$.

(a) Problem (5) is equivalent to $B u=\ell$ in $\mathcal{H}^{*}$. As $\operatorname{im} B \cong \mathcal{H}^{*} / \operatorname{ker} B^{*} \cong\left(\operatorname{ker} B^{*}\right)^{\perp}$, we deduce that $B u=\ell$ has a solution $u \in \mathcal{H}$ if and only if $\ell \in\left(\operatorname{ker} B^{*}\right)^{\perp}$. We are hence left to prove that $\operatorname{ker} B^{*}=\operatorname{ker}\left(b^{*}\right)$. Using the definition of $B^{*}$, we deduce that

$$
b(u, v)=\langle B u, v\rangle_{\mathcal{H}^{*}}=\left\langle u, B^{*} v\right\rangle_{\mathcal{H}}={\overline{\left\langle B^{*} v, u\right\rangle_{\mathcal{H}}}}
$$

for all $u, v \in \mathcal{H}$. Hence, by identifying $\mathcal{H}$ with $\mathcal{H}^{*}$, we find that $B^{*} v=b^{*}(v, \cdot)$ for all $v \in \mathcal{H}$ and consequently $\operatorname{ker} B^{*}=\operatorname{ker}\left(b^{*}\right)$. This proves the claim.

(b) As ind $B=0$, the adjoint $B^{*}$ is also a Fredholm operator of index 0 . Hence $\operatorname{dim} \operatorname{ker} B=\operatorname{dim} \operatorname{coker} B=\operatorname{dim} \operatorname{ker} B^{*}$. Thus $\operatorname{dim} \operatorname{ker}(b)=\operatorname{dim} \operatorname{ker} B=\operatorname{dim} \operatorname{ker} B^{*}=$ $\operatorname{dim} \operatorname{ker}\left(b^{*}\right)$. Finiteness of the dimensions follows since $B$ is Fredholm operator.

(c) This statement is an immediate consequence of $(a)$ and $(b)$.

\subsection{Limiting absorption principle for sesquilinear forms}

Given $\ell \in \mathcal{H}^{*}$, a standard tool to prove the existence of a solution $u \in \mathcal{H}$ to

$$
b(u, \cdot)=\ell(\cdot)
$$

is the Lax-Milgram lemma. For this tool to work, $b$ has to be coercive. If $b$ is not coercive, one can replace it by a family of suitable sesquilinear forms $\left(b_{\delta}\right)_{\delta}$ and prove, for each $\delta>0$, the existence of a solution $u_{\delta} \in \mathcal{H}$ to

$$
b_{\delta}\left(u_{\delta}, \cdot\right)=\ell(\cdot) .
$$

The idea is then to show that the solutions $\left(u_{\delta}\right)_{\delta}$ of (7) converge in $\mathcal{H}$ to a solution of (6) as $\delta \rightarrow 0$.

We provide a sufficient condition for this method to work.

Assumption 2.1 The sesquilinear form $b: \mathcal{H} \times \mathcal{H} \rightarrow \mathbb{K}$ satisfies the following two requirements:

(i) (Uniqueness) The kernel of $b$ is trivial.

(ii) (Gårding) There exist a constant $c>0$ and a compact operator $K: \mathcal{H} \rightarrow \mathcal{H}^{*}$ such that

$$
\left|b(u, u)+\langle K u, u\rangle_{\mathcal{H}^{*}, \mathcal{H}}\right| \geq c\|u\|^{2} \quad \text { for all } u \in \mathcal{H} .
$$

We observe that every coercive sesquilinear form satisfies Assumption 2.1.

Lemma 2.2 Let $\mathcal{H}$ be a Hilbert space and let $b: \mathcal{H} \times \mathcal{H} \rightarrow \mathbb{K}$ be a bounded sesquilinear form that satisfies Assumption 2.1. Then, for every $\ell \in \mathcal{H}^{*}$ there is a unique $u \in \mathcal{H}$ such that 


$$
b(u, \cdot)=\ell(\cdot) .
$$

Proof The claim follows by applying Proposition 2.1(c).

Theorem 2.1 (Abstract limiting absorption principle) Let $\mathcal{H}$ be a Hilbert space over the field $\mathbb{K}$ and let $b: \mathcal{H} \times \mathcal{H} \rightarrow \mathbb{K}$ be a bounded sesquilinear form. Assume that $\left(b_{\delta}\right)_{\delta}$ is a family of bounded sesquilinear forms that satisfies the following requirements:

(a) For each $\delta>0$, the sesquilinear form $b_{\delta}$ satisfies Assumption 2.1.

(b) For $u, v \in \mathcal{H}$ fixed, $\lim _{\delta \rightarrow 0} b_{\delta}(u, v)=b(u, v)$.

(c) For every sequence $\left(u_{\delta}\right)_{\delta}$ in $\mathcal{H}$ with $u_{\delta} \rightarrow u$ weakly in $\mathcal{H}$ there holds:

$$
\lim _{\delta \rightarrow 0} b_{\delta}\left(u_{\delta}-u, v\right)=0 \quad \text { for all } v \in \mathcal{H} .
$$

Assume further that

$$
\operatorname{ker}(b)=\{0\} .
$$

Then the following holds: For every $\ell \in \mathcal{H}^{*}$ there exists a sequence $\left(u_{\delta}\right)_{\delta}$ such that $b_{\delta}\left(u_{\delta}, \cdot\right)=\ell(\cdot)$, for each $\delta>0$. The sequence $\left(u_{\delta}\right)_{\delta}$ weakly converges in $\mathcal{H}$ to an element $u \in \mathcal{H}$ satisfying

$$
b(u, \cdot)=\ell(\cdot) .
$$

Moreover, the limit $u$ is the unique solution to (8).

Proof Fix $\ell \in \mathcal{H}^{*}$. Lemma 2.2 together with assumption (a) imply that, for each $\delta>0$, there is a unique solution $u_{\delta} \in \mathcal{H}$ to $b_{\delta}\left(u_{\delta}, \cdot\right)=\ell(\cdot)$.

Case 1. Assume that $\left(u_{\delta}\right)_{\delta}$ is bounded in $\mathcal{H}$. We then find a subsequence $\left(u_{\delta}\right)_{\delta}$ and an element $u \in \mathcal{H}$ such that $u_{\delta} \rightarrow u$ weakly in $\mathcal{H}$. For all $v \in \mathcal{H}$ there holds

$$
\left|b_{\delta}\left(u_{\delta}, v\right)-b(u, v)\right| \leq\left|b_{\delta}\left(u_{\delta}-u, v\right)\right|+\left|b_{\delta}(u, v)-b(u, v)\right| .
$$

Thanks to $(b)$ and $(c)$, we conclude from (9) that

$$
\lim _{\delta \rightarrow 0} b_{\delta}\left(u_{\delta}, v\right)=b(u, v) \quad \text { for all } v \in \mathcal{H} .
$$

On the other hand, for each $\delta>0$, we have that $b_{\delta}\left(u_{\delta}, \cdot\right)=\ell(\cdot)$. Combining this with (10) we infer that $b(u, v)=\ell(v)$ for all $v \in \mathcal{H}$ and a solution $u \in \mathcal{H}$ is found. Uniqueness of $u$ follows as $\operatorname{ker}(b)=\{0\}$. As the limit $u$ is unique, we infer that every subsequence of the bounded sequence $\left(u_{\delta}\right)_{\delta}$ has a subsequence that weakly converges in $\mathcal{H}$ to $u$, and thus the whole sequence converges weakly.

Case 2. Suppose that $\left(u_{\delta}\right)_{\delta}$ is unbounded in $\mathcal{H}$. We shall prove that this is impossible. As $\left(u_{\delta}\right)_{\delta}$ is unbounded, we find a subsequence $\left(u_{\delta}\right)_{\delta}$ with $\left\|u_{\delta}\right\| \rightarrow \infty$ as $\delta \rightarrow 0$. Consider the re-scaled sequence $\left(w_{\delta}\right)_{\delta}$ given by

$$
w_{\delta}:=\frac{u_{\delta}}{\left\|u_{\delta}\right\|} \in \mathcal{H} .
$$

Clearly, $\left(w_{\delta}\right)_{\delta}$ is bounded in $\mathcal{H}$ and thus there is an element $w \in \mathcal{H}$ such that $w_{\delta} \rightarrow w$ weakly in $\mathcal{H}$. For each $\delta>0$, the function $w_{\delta} \in \mathcal{H}$ satisfies the equation 


$$
b_{\delta}\left(w_{\delta}, v\right)=\frac{\ell(v)}{\left\|u_{\delta}\right\|} \quad \text { for all } v \in \mathcal{H} .
$$

As the sequence $\left(u_{\delta}\right)_{\delta}$ is unbounded in $\mathcal{H}$, we deduce from $(b),(c)$, and equation (11) that

$$
b(w, v)=\lim _{\delta \rightarrow 0} b_{\delta}\left(w_{\delta}, v\right)=\lim _{\delta \rightarrow 0} \frac{\ell(v)}{\left\|u_{\delta}\right\|}=0 \quad \text { for all } v \in \mathcal{H} .
$$

By assumption $\operatorname{ker}(b)=\{0\}$ and thus $w=0$.

As $\ell$ is bounded, we deduce from (11) that $\lim _{\delta \rightarrow 0} b_{\delta}\left(w_{\delta}, w_{\delta}\right)=0$. The sesquilinear forms $\left(b_{\delta}\right)_{\delta}$ satisfy Assumption 2.1; in particular, a Gårding inequality holds. Consequently, there exist a compact operator $K: \mathcal{H} \rightarrow \mathcal{H}^{*}$ and a constant $c>0$ such that

$$
0<c=c\left\|w_{\delta}\right\|^{2} \leq\left|b_{\delta}\left(w_{\delta}, w_{\delta}\right)\right|+\left|\left\langle K w_{\delta}, w_{\delta}\right\rangle\right| .
$$

Sending $\delta \rightarrow 0$ in (12), we find that

$$
0<c \leq|\langle K w, w\rangle|
$$

This is a contradiction and hence the sequence $\left(u_{\delta}\right)_{\delta}$ cannot be unbounded in $\mathcal{H}$.

\section{Existence result for a periodic bounded waveguide}

In this section we apply Theorem 2.1 to a Helmholtz equation in a bounded waveguide, and extend the analytical results of [4].

\subsection{Setting}

Let us describe the setting of [4]: Fix a periodicity $\varepsilon>0$, and define the domain $\Omega_{R}:=(-R \varepsilon, R \varepsilon) \times(0, \varepsilon H)$ with $R>0$ and $H \in \mathbb{N}$. Assume that $a_{+}, a_{-}: \mathbb{R}^{2} \rightarrow \mathbb{R}$ are two $\varepsilon$-periodic functions for which there exist constants $c_{2}>c_{1}>0$ such that $c_{1} \leq a_{+}, a_{-} \leq c_{2}$. Define $a: \mathbb{R}^{2} \rightarrow \mathbb{R}$ by

$$
a(x):= \begin{cases}a_{+}(x) & \text { for } x \in[0, \infty) \times \mathbb{R}, \\ a_{-}(x) & \text { for } x \in(-\infty, 0) \times \mathbb{R} .\end{cases}
$$

Fix $\delta, R, L>0$ and $f \in L^{2}\left(\Omega_{R}\right)$. Below, we will construct functions $u_{\delta}: \Omega_{R+L} \rightarrow$ $\mathbb{C}$ that satisfy the Helmholtz equation with absorption parameter $\delta>0$,

$$
-\nabla \cdot\left(a \nabla u_{\delta}\right)-\omega^{2}(1+\mathrm{i} \delta) u_{\delta}=f \quad \text { in } \Omega_{R},
$$

and that are periodic in vertical direction. Furthermore, we construct a vertically periodic function $u: \Omega_{R+L} \rightarrow \mathbb{C}$ satisfying 


$$
-\nabla \cdot(a \nabla u)-\omega^{2} u=f \quad \text { in } \Omega_{R} .
$$

To formulate the radiation conditions, we introduce radiation boxes

$$
W_{R, L}^{-}:=(-\varepsilon(R+L),-\varepsilon R) \times(0, \varepsilon H) \quad \text { and } \quad W_{R, L}^{+}:=(\varepsilon R, \varepsilon(R+L)) \times(0, \varepsilon H) \text {. }
$$

The restriction of $u: \Omega_{R+L} \rightarrow \mathbb{R}$ to $W_{R, L}^{ \pm}$is denoted by $\mathcal{R}_{R, L}^{ \pm}(u)$. More precisely, we also shift in the parameter $x_{1}$ and set $\mathcal{R}_{R, L}^{-}(u), \mathcal{R}_{R, L}^{+}(u):[0, \varepsilon L) \times[0, \varepsilon H) \rightarrow \mathbb{R}$,

$$
\mathcal{R}_{R, L}^{-}(u)\left(x_{1}, x_{2}\right):=u\left(-\varepsilon(R+L)+x_{1}, x_{2}\right) \text { and } \mathcal{R}_{R, L}^{+}(u)\left(x_{1}, x_{2}\right):=u\left(\varepsilon R+x_{1}, x_{2}\right) \text {. }
$$

Roughly speaking, the radiation condition at infinity is replaced by the requirement that the solution $u$ of (14) should be a right-going wave in $W_{R, L}^{+}$and a left-going wave in $W_{R, L}^{-}$. To make this idea precise, we need the Bloch formalism.

Bloch expansion. Set $Y_{\varepsilon}:=\varepsilon(0,1)^{2}$. For fixed $N, M \in \mathbb{N}$ we consider the index sets

$$
Q_{N}:=\left\{0, \frac{1}{N}, \ldots, \frac{N-1}{N}\right\}
$$

and

$$
I_{N, M}:=\left\{(j, m): j \in Q_{N} \times Q_{M} \text { and } m \in \mathbb{N}_{0}\right\} .
$$
by

For each $j \in Q_{N} \times Q_{M}$ we consider $\mathcal{L}_{j}^{+}, \mathcal{L}_{j}^{-}: H_{\text {per }}^{1}\left(Y_{\varepsilon} ; \mathbb{C}\right) \rightarrow\left(H_{\text {per }}^{1}\left(Y_{\varepsilon} ; \mathbb{C}\right)\right)^{*}$ given

$$
\mathcal{L}_{j}^{+}:=-(\nabla+2 \pi \mathrm{i} j / \varepsilon) \cdot\left[a_{+}(x)(\nabla+2 \pi \mathrm{i} j / \varepsilon)\right],
$$

and

$$
\mathcal{L}_{j}^{-}:=-(\nabla+2 \pi \mathrm{i} j / \varepsilon) \cdot\left[a_{-}(x)(\nabla+2 \pi \mathrm{i} j / \varepsilon)\right] .
$$

The definition of these differential operators is motivated by the following observation: If $\psi_{j}^{ \pm}$is an eigenfunction of $\mathcal{L}_{j}^{ \pm}$with eigenvalue $\mu_{j}^{ \pm}$, then $x \mapsto \psi_{j}^{ \pm}(x) \mathrm{e}^{2 \pi \mathrm{i} j \cdot x / \varepsilon}$ is a solution to the Helmholtz equation (15) with $a=a_{ \pm}, \omega^{2}=\mu_{j}^{ \pm}$, and $f=0$.

Let $j \in[0,1]^{2}$ be a fixed wave vector. We denote by $\left(\psi_{j, m}^{+}\right)_{m \in \mathbb{N}_{0}}$ a family of eigenfunctions of the differential operator $\mathcal{L}_{j}^{+}$. The labelling is in such a way that the corresponding eigenvalues $\left(\mu_{m}^{+}(j)\right)_{m}$ satisfy $\mu_{m+1}^{+}(j) \geq \mu_{m}^{+}(j)$ for all $m \in \mathbb{N}_{0}$. Similarly, the eigenfunctions of $\mathcal{L}_{j}^{-}$are denoted by $\left(\psi_{j, m}^{-}\right)_{m \in \mathbb{N}_{0}}$. We normalise with the condition

$$
f_{Y_{\varepsilon}}\left|\psi_{j, m}^{ \pm}\right|^{2}=1 \quad \text { for all } m \in \mathbb{N}_{0}
$$

The eigenfunctions $\left(\psi_{j, m}^{ \pm}\right)_{m \in \mathbb{N}}$ are called Bloch eigenfunctions. The following expansion is classical; see, for instance, [1, Lemma 4.9].

Lemma 3.1 (Bloch expansion) For $L, H \in \mathbb{N}$ and $\varepsilon>0$ denote by $W_{\varepsilon}$ the rectangle $(0, \varepsilon L) \times(0, \varepsilon H)$. Let $u \in L^{2}\left(W_{\varepsilon} ; \mathbb{C}\right)$. Then for both families of eigenfunctions $\left(\psi_{j, m}^{+}\right)_{m}$ and $\left(\psi_{j, m}^{-}\right)_{m}$, the function $u$ admits a unique expansion

$$
u(x)=\sum_{(j, m) \in I_{N, M}} \alpha_{j, m}^{ \pm} \psi_{j, m}^{ \pm}(x) \mathrm{e}^{2 \pi \mathrm{i} j \cdot x / \varepsilon} \quad \text { in } L^{2}\left(W_{\varepsilon}, \mathbb{C}\right),
$$


where $\alpha_{j, m}^{ \pm} \in \mathbb{C}$ for all $(j, m) \in I_{N, M}$.

To shorten the notation, let us introduce for $\lambda=(j, m) \in I_{N, M}$ the functions

$$
U_{\lambda}: W_{\varepsilon} \rightarrow \mathbb{R}, \quad U_{\lambda}^{ \pm}(x):=\psi_{\lambda}^{ \pm}(x) \mathrm{e}^{2 \pi \mathrm{i} j \cdot x / \varepsilon} .
$$

Having the families $\left(U_{\lambda}^{+}\right)_{\lambda}$ and $\left(U_{\lambda}^{-}\right)_{\lambda}$, the expansion (16) reads

$$
u=\sum_{\lambda \in I_{N, M}} \alpha_{\lambda}^{ \pm} U_{\lambda}^{ \pm} \quad \text { in } L^{2}\left(W_{\varepsilon} ; \mathbb{C}\right),
$$

where $\alpha_{\lambda}^{ \pm} \in \mathbb{C}$ for all $\lambda \in I_{N, M}$.

We recall from [1] the following Plancherel formula

$$
\|u\|_{L^{2}\left(W_{\varepsilon} ; \mathbb{C}\right)}^{2}=\varepsilon^{2} L H \sum_{\lambda \in I_{N, M}}\left|\alpha_{\lambda}^{ \pm}\right|^{2} .
$$

Radiation condition in a bounded waveguide. The Bloch eigenfunctions $\left(U_{\lambda}^{+}\right)_{\lambda}$ and $\left(U_{\lambda}^{-}\right)_{\lambda}$ may transport energy in any direction. To indicate in which direction along the $x_{1}$-axis energy is transported, we introduce the Poynting numbers

$$
P_{\lambda}^{ \pm}:=\operatorname{Im} f_{Y_{\varepsilon}} \bar{U}_{\lambda}^{ \pm}(x) e_{1} \cdot\left[a_{ \pm}(x) \nabla U_{\lambda}^{ \pm}(x)\right] \mathrm{d} x .
$$

For $N, M \in \mathbb{N}$, let us define two index sets

$$
I_{N, M}^{-} \subset\left\{\lambda \in I_{N, M}: P_{\lambda}^{-}<0\right\} \quad \text { and } \quad I_{N, M}^{+} \subset\left\{\lambda \in I_{N, M}: P_{\lambda}^{+}>0\right\},
$$

and the function spaces

$$
X_{\leftarrow}^{-}:=\operatorname{span}\left\{U_{\lambda}^{-}: \lambda \in I_{N, M}^{-}\right\} \quad \text { and } \quad X_{\rightarrow}^{+}:=\operatorname{span}\left\{U_{\lambda}^{+}: \lambda \in I_{N, M}^{+}\right\} .
$$

We note that $X_{\leftarrow}^{-}$is the space spanned by all left-going Bloch eigenfunctions $U_{\lambda}^{-}$ which are defined on the radiation box $W_{R, L}^{-}$, and $X_{\rightarrow}^{+}$is the space spanned by all right-going Bloch eigenfunctions $U_{\lambda}^{+}$defined on $W_{R, L}^{+}$.

The function space in which we look for a solution to (14) is

$$
V_{R}:=\left\{u \in H^{1}\left(\Omega_{R+L}\right): \begin{array}{l}
u\left(x_{1}, \cdot\right) \text { is periodic for almost all } x_{1} \in(-R, R), \\
\mathcal{R}_{R, L}^{-}(u) \in X_{\leftarrow}^{-} \text {and } \mathcal{R}_{R, L}^{+}(u) \in X_{\rightarrow}^{+}
\end{array}\right\} .
$$

The variational formulation. For a weak formulation of (14) and (15), we introduce the sesquilinear forms $Q_{+}, Q_{-}, Q: V_{R} \times V_{R} \rightarrow \mathbb{C}$,

$$
Q_{+}(u, v):=\frac{1}{\varepsilon L} \int_{W_{R, L}^{+}} \bar{u} e_{1} \cdot a_{+} \nabla v, \quad Q_{-}(u, v):=\frac{1}{\varepsilon L} \int_{W_{R, L}^{-}} \bar{u} e_{1} \cdot a_{-} \nabla v,
$$

and

$$
Q(u, v):=Q_{+}(u, v)-Q_{-}(u, v)
$$


which we will use to encode the radiation conditions. We further define the function $\vartheta: \Omega_{R+L} \rightarrow[0,1]$

$$
\vartheta\left(x_{1}, x_{2}\right):= \begin{cases}0 & \text { for }\left|x_{1}\right| \geq R+L, \\ 1 & \text { for }\left|x_{1}\right| \leq R, \\ \frac{1}{L}\left(R+L-\left|x_{1}\right|\right) & \text { otherwise }\end{cases}
$$

For $R, L, \omega^{2}>0$ and $\delta \geq 0$, we define the sesquilinear form $\beta_{\delta}: V_{R} \times V_{R} \rightarrow \mathbb{C}$ by

$$
\beta_{\delta}(u, v):=\int_{\Omega_{R+L}} \vartheta a \nabla \bar{u} \cdot \nabla v-\omega^{2}(1-\mathrm{i} \delta) \int_{\Omega_{R+L}} \bar{u} v \vartheta+Q(u, v),
$$

where the coefficient $a: \Omega_{R+L} \rightarrow \mathbb{R}$ is given in (13).

The following problem was introduced in in [4].

Definition 3.1 (Solution concept) We say that $u: \Omega_{R+L} \rightarrow \mathbb{C}$ solves the truncated radiation problem with absorption if it is an element of $V_{R}$ and satisfies

$$
\beta_{\delta}(u, v)=\int_{\Omega_{R}} \bar{f} v
$$

for all $v \in V_{R}$. If $u$ satisfies (25) with $\delta=0$, then $u$ is a solution to the truncated radiation problem.

\subsection{Existence result for the Helmholtz equation with damping}

We need the following assumptions to prove the existence of solutions to the truncated radiation problem.

Assumption 3.1 Let $I_{N, M}^{-}$and $I_{N, M}^{+}$be the index sets of (18), and let $X_{\leftarrow}^{-}$and $X_{\rightarrow}^{+}$ be the function spaces of (19).

(A1) Every pair of indices $\lambda=(j, m)$ and $\tilde{\lambda}=(\tilde{j}, \tilde{m})$ in $I_{N, M}^{-}$with $\lambda \neq \tilde{\lambda}$ satisfies $j \neq \tilde{j}$. The same holds for $I_{N, M}^{+}$.

(A2) There exists $C_{0}>0$ such that for all $u \in X_{\leftarrow}^{-}$and $v \in X_{\rightarrow}^{+}$there holds

$$
\|u\|_{H^{1}\left(W_{R, L}^{-}\right)} \leq C_{0}\|u\|_{L^{2}\left(W_{R, L}^{-}\right)} \text {and }\|v\|_{H^{1}\left(W_{R, L}^{+}\right)} \leq C_{0}\|v\|_{L^{2}\left(W_{R, L}^{+}\right)} .
$$

We note that every $\lambda \in I_{N, M}^{-}$is a pair $(j, m)$ with $j \in Q_{N} \times Q_{M}$ and $m \in \mathbb{N}$, where $Q_{N}$ and $Q_{M}$ are finite index sets. Thus, Assumption (A1) implies that both index sets $I_{N, M}^{-}$and $I_{N, M}^{+}$are finite. This ensures, in particular, that the function spaces $X_{\leftarrow}^{-}$and $X_{\rightarrow}^{+}$are finite dimensional and hence closed. $V_{R} \subset H^{1}\left(\Omega_{R+L}\right)$ is thus a Hilbert space. Moreover, as both $X_{\leftarrow}^{-}$and $X_{\rightarrow}^{+}$are finite dimensional, we can expect a regularity property as in (A2). A consequence of (A2) is a Gårding inequality. For such a Gårding inequality we need a compact operator. Define $K: V_{R} \rightarrow V_{R}^{*}$, $u \mapsto K u$, by 


$$
\langle K u, v\rangle_{V_{R}^{*}, V_{R}}:=2 \omega^{2}\langle u, v\rangle_{L^{2}\left(\Omega_{R+L}\right)}-\mathrm{i} \delta \omega^{2}\langle u, \vartheta v\rangle_{L^{2}\left(\Omega_{R+L}\right)}-Q(u, v),
$$

where $Q$ is the sesquilinear form defined in (22). In this definition of $K$ we allow $\delta \geq 0$. We first show that $K$ is indeed compact.

Lemma 3.2 (Compactness of $K$ ) Let $R, L$, and $\omega^{2}$ be positive numbers and $\delta \geq 0$. Let $V_{R}$ be the function space defined in (20) and let Assumption 3.1 hold. Then the operator $K$ defined in (27) is compact.

Proof For brevity we write $L^{2}$ instead of $L^{2}\left(\Omega_{R+L}\right)$. The compactness of $K: V_{R} \rightarrow$ $V_{R}^{*}$ is equivalent to the following condition: Every bounded sequence $\left(u_{m}\right)_{m}$ in $V_{R}$ admits a subsequence $\left(u_{m}\right)_{m}$ that weakly converges to some $u \in V_{R}$ and satisfies

$$
\left\langle K u_{m}, v_{m}\right\rangle_{V_{R}^{*}, V_{R}} \rightarrow\langle K u, v\rangle_{V_{R}^{*}, V_{R}} \quad \text { as } m \rightarrow \infty
$$

for all sequences $\left(v_{m}\right)_{m}$ in $V_{R}$ with $v_{m} \rightarrow v$ weakly in $V_{R}$.

Let $\left(u_{m}\right)_{m}$ and $\left(v_{m}\right)_{m}$ be sequences in $V_{R}$ such that $\left(u_{m}\right)_{m}$ is bounded and $\left(v_{m}\right)_{m}$ weakly converges in $V_{R}$ to $v \in V_{R}$. As $V_{R}$ is a Hilbert space, $\left(u_{m}\right)_{m}$ admits a subsequence $\left(u_{m}\right)_{m}$ that weakly converges to $u \in V_{R}$. The function space $V_{R}$ is a subspace of $H^{1}\left(\Omega_{R+L}\right)$ and thus, by the Rellich-Kondrachov theorem, $u_{m} \rightarrow u$ in $L^{2}$. Moreover, the weak convergence of $\left(v_{m}\right)_{m}$ in $V_{R}$ implies that $v_{m} \rightarrow v$ weakly in $L^{2}$ as well as $\nabla v_{m} \rightarrow \nabla v$ weakly in $L^{2}$. Using the definition of $Q$ in (22), the strong $L^{2}$-convergence of $\left(u_{m}\right)_{m}$ and the weak $L^{2}$-convergence of $\left(\nabla v_{m}\right)_{m}$, we deduce that $Q\left(u_{m}, v_{m}\right) \rightarrow Q(u, v)$ as $m \rightarrow \infty$. Consequently,

$$
\begin{aligned}
\lim _{m \rightarrow \infty}\left\langle K u_{m}, v_{m}\right\rangle_{V_{R}^{*}, V_{R}} & =\lim _{m \rightarrow \infty}\left(2 \omega^{2}\left\langle u_{m}, v_{m}\right\rangle_{L^{2}}-\mathrm{i} \delta \omega^{2}\left\langle u_{m}, \vartheta v_{m}\right\rangle_{L^{2}}-Q\left(u_{m}, v_{m}\right)\right) \\
& =2 \omega^{2}\langle u, v\rangle_{L^{2}}-\mathrm{i} \delta \omega^{2}\langle u, \vartheta v\rangle_{L^{2}}-Q(u, v) \\
& =\langle K u, v\rangle_{V_{R}^{*}, V_{R}} .
\end{aligned}
$$

This proves the claim.

Lemma 3.3 (Gårding inequality) Let $R, L$, and $\omega^{2}$ be positive numbers, and let $V_{R}$ be the function space given in (20). Suppose that Assumption 3.1 holds. For each $\delta \geq 0$, the sesquilinear form $\beta_{\delta}$ satisfies a Gårding inequality. More precisely, there exists a constant $c>0$ such that for all $u \in V_{R}$ there holds

$$
\left|\beta_{\delta}(u, u)+\langle K u, u\rangle_{V_{R}^{*}, V_{R}}\right| \geq c\|u\|_{H^{1}\left(\Omega_{R+L}\right)}^{2},
$$

where $K: V_{R} \rightarrow V_{R}^{*}$ is the operator defined in (27).

Proof Fix $u \in V_{R}$. Using the definition of $K$, we find that

$$
\beta_{\delta}(u, u)+\langle K u, u\rangle_{V_{R}^{*}, V_{R}}=\int_{\Omega_{R+L}} \vartheta a|\nabla u|^{2}+\omega^{2} \int_{\Omega_{R+L}}(2-\vartheta)|u|^{2} .
$$

Equation (28) and Assumption 3.1 (A2) imply that 


$$
\begin{aligned}
\left|\beta_{\delta}(u, u)+\langle K u, u\rangle_{V_{R}^{*}, V_{R}}\right| & \geq c_{1}\|\nabla u\|_{L^{2}\left(\Omega_{R}\right)}^{2}+\omega^{2}\|u\|_{L^{2}\left(\Omega_{R+L}\right)}^{2} \\
& \geq \min \left\{c_{1}, \omega^{2}\right\}\|u\|_{H^{1}\left(\Omega_{R}\right)}^{2}+\omega^{2} C_{0}^{-1}\|u\|_{H^{1}\left(W_{R, L}^{-} \cup W_{R, L}^{+}\right)}^{2} \\
& =C_{1}\|u\|_{H^{1}\left(\Omega_{R+L}\right)}^{2} .
\end{aligned}
$$

Thanks to Lemma 3.2 we know that $K$ is compact. This together with (29) proves the claim.

Lemma 3.4 (Uniqueness of the homogeneous problem with $\delta>0$ ) Let $R, L$, and $\omega^{2}$ be positive numbers, and let $V_{R}$ be the function space defined in (20). Fix $\delta>0$. If $u \in V_{R}$ satisfies $\beta_{\delta}(u, \cdot)=0$, then $u=0$.

Proof Suppose that there is an element $u \in V_{R}$ with $u \neq 0$ and $\beta_{\delta}(u, v)=0$ for all $v \in V_{R}$. Taking the imaginary part of $\beta_{\delta}(u, u)$, we find that

$$
\omega^{2} \delta \int_{\Omega_{R+L}}|u|^{2} \vartheta+\operatorname{Im} Q(u, u)=0 .
$$

We first show that both terms are non-negative. By definition of the function space $V_{R}$, the function $u$ can be expanded in Bloch eigenfunctions in the radiation boxes $W_{R, L}^{-}$and $W_{R, L}^{+}$,

$$
\mathcal{R}_{R, L}^{-}(u)=\sum_{\lambda \in I_{N, M}^{-}} \alpha_{\lambda}^{-} U_{\lambda}^{-} \quad \text { and } \quad \mathcal{R}_{R, L}^{+}(u)=\sum_{\lambda \in I_{N, M}^{+},} \alpha_{\lambda}^{+} U_{\lambda}^{+},
$$

where $\alpha_{\lambda}^{ \pm} \in \mathbb{C}$ for all $\lambda \in I_{N, M}^{ \pm}$. The Bloch eigenfunctions $\left(U_{\lambda}^{ \pm}\right)$are orthogonal with respect to $Q_{ \pm}$, that is,

$$
Q_{ \pm}\left(U_{\lambda}^{ \pm}, U_{\mu}^{ \pm}\right)=0 \quad \text { for all } \lambda, \mu \in I_{N, M}^{ \pm} \text {with } \lambda \neq \mu .
$$

We refer to Lemma 2.4 in [4] for a proof of this orthogonality property. Using the expansion (31) and the orthogonality property (32), we compute that

$$
\begin{aligned}
Q(u, u) & =Q_{+}\left(\mathcal{R}_{R, L}^{+}(u), \mathcal{R}_{R, L}^{+}(u)\right)-Q_{-}\left(\mathcal{R}_{R, L}^{-}(u), \mathcal{R}_{R, L}^{-}(u)\right) \\
& =\sum_{\lambda \in I_{N, M}^{+},}\left|\alpha_{\lambda}^{+}\right|^{2} Q_{+}\left(U_{\lambda}^{+}, U_{\lambda}^{+}\right)-\sum_{\lambda \in I_{N, M}^{-}}\left|\alpha_{\lambda}^{-}\right|^{2} Q_{-}\left(U_{\lambda}^{-}, U_{\lambda}^{-}\right) .
\end{aligned}
$$

Using the definition of the Poynting numbers in (17), and the definition of the index sets $I_{N, M}^{-}$and $I_{N, M}^{+}$, we infer from (33) that

$$
\operatorname{Im} Q(u, u)=\sum_{\lambda \in I_{N, M}^{+}}\left|\alpha_{\lambda}^{+}\right|^{2} P_{\lambda}^{+}-\sum_{\lambda \in I_{N, M}^{-}}\left|\alpha_{\lambda}^{-}\right|^{2} P_{\lambda}^{-} \geq 0 .
$$

By assumption $u \neq 0$ in $\Omega_{R+L}$. If $u \neq 0$ in $\Omega_{R}$, then the first term on the left-hand side of (30) is positive and the second is non-negative, which is a contradiction with the right-hand side. On the other hand, if $u \neq 0$ in $W_{R}^{-}$or $u \neq 0$ in $W_{R}^{+}$, then 
$\operatorname{Im} Q(u, u)>0$. But this contradicts equation (30) as well. Consequently, $u=0$ almost everywhere in $\Omega_{R+L}$ and the claim is proved.

Theorem 3.1 (Existence result for $\delta>0$ ) Let $R, L$, and $\omega^{2}$ be positive numbers, $f \in L^{2}\left(\Omega_{R}\right)$, and let $a: \Omega_{R+L} \rightarrow \mathbb{R}$ be as in (13). Let $V_{R}$ be the function space given in (20) and assume that Assumption 3.1 is satisfied. If $\delta>0$, then there exists a unique solution $u \in V_{R}$ to the truncated radiation problem. More precisely, for every $v \in V_{R}$ there holds

$$
\beta_{\delta}(u, v)=\int_{\Omega_{R}} \bar{f} v,
$$

where $\beta_{\delta}$ is the sesquilinear form defined in (24).

Proof Thanks to Assumption 3.1 (A1), $V_{R}$ is a Hilbert space. The sesquilinear form $\beta_{\delta}$ is bounded and satisfies a Garding inequality by Lemma 3.3. Hence, the claim follows from Lemma 2.2 as soon as we show that the only element $w \in V_{R}$ that satisfies $\beta_{\delta}(w, v)=0$ for all $v \in V_{R}$ is $w=0$. But this is true due to Lemma 3.4.

Remark 3.1 The above existence result was also proved in [4]. There, however, the authors had to make an additional assumption (besides Assumption 3.1):

(A3) There exists a positive number $c_{0}>0$ such that $P_{\lambda}^{+} \geq c_{0}$ for every $\lambda \in I_{N, M}^{+}$. Furthermore, for every $\lambda \in I_{N, M}^{-}$there holds $-P_{\lambda}^{-} \geq c_{0}$.

Here $P_{\lambda}^{ \pm}$are the Poynting numbers defined in (17). This additional assumption is needed to show coercivity of the sesquilinear form $\beta_{\delta}$ from which existence of a solution to the truncated radiation problem follows. Here, on the other hand, we deduce existence from a Gårding inequality and hence do not have to prove coercivity of $\beta_{\delta}$. Let us remark, however, that (A3) is satisfied since assumption (A1) implies that the index sets $I_{N, M}^{ \pm}$are finite.

\subsection{Existence result for the Helmholtz equation}

In this section, we apply the limiting absorption principle to prove the existence of a solution to the truncated radiation problem. We recall that, by Definition 3.1, a function $u \in V_{R}$ is a solution provided

$$
\beta(u, v)=\int_{\Omega_{R}} \bar{f} v \quad \text { for all } v \in V_{R},
$$

where the sesquilinear form $\beta: V_{R} \times V_{R} \rightarrow \mathbb{C}$ is defined as

$$
\beta(u, v):=\int_{\Omega_{R+L}} \vartheta a \nabla \bar{u} \cdot \nabla v-\omega^{2} \int_{\Omega_{R+L}} \bar{u} v \vartheta+Q(u, v) .
$$

Theorem 3.2 (Limiting absorption principle) Let $R, L$ and $\omega^{2}$ be positive numbers, $f \in L^{2}\left(\Omega_{R}\right)$, and let $a: \Omega_{R+L} \rightarrow \mathbb{R}$ be as in (13). Let $V_{R}$ be the function space given 
in (20) and let Assumption 3.1 hold. Assume further that $\operatorname{ker}(\beta)=\{0\}$. Then for every $f \in L^{2}\left(\Omega_{R}\right)$ there exists a sequence $\left(u_{\delta}\right)_{\delta}$ of solutions to the truncated radiation problem that weakly converges in $V_{R}$ to a function $u \in V_{R}$ satisfying

$$
\beta(u, v)=\int_{\Omega_{R}} \bar{f} v \quad \text { for all } v \in V_{R} .
$$

Moreover, this limit u is the unique solution to (35).

Proof We use the limiting absorption principle of Theorem 2.1. By Assumption 3.1 (A1), $V_{R}$ is a Hilbert space. For each $\delta>0$, the sesquilinear form $\beta_{\delta}$ defined in (24) is bounded. One readily checks that $\lim _{\delta \rightarrow 0} \beta_{\delta}(u, v)=\beta(u, v)$ for all $u, v \in V_{R}$. We are thus left to prove that premises $(a)$ and $(c)$ of Theorem 2.1 hold. Lemmas 3.3 and 3.4 ensure that $(a)$ is satisfied.

Let $\left(u_{\delta}\right)_{\delta}$ be a sequence in $V_{R}$ with $u_{\delta} \rightarrow u$ weakly in $V_{R}$. Thanks to the RellichKondrachov theorem, we find a subsequence $\left(u_{\delta}\right)_{\delta}$ that strongly converges to $u$ in $L^{2}\left(\Omega_{R+L}\right)$. Thus, for every $v \in V_{R}$, we have that

$$
\lim _{\delta \rightarrow 0}\left[\omega^{2}(1+\mathrm{i} \delta) \int_{\Omega_{R+L}} \vartheta\left(\bar{u}_{\delta}-\bar{u}\right) v\right]=0 \quad \text { and } \quad \lim _{\delta \rightarrow 0} Q\left(u_{\delta}-u, v\right)=0 .
$$

On the other hand, since $\nabla u_{\delta} \rightarrow \nabla u$ weakly in $L^{2}\left(\Omega_{R+L}\right)$ as $\delta \rightarrow 0$, we conclude that

$$
\lim _{\delta \rightarrow 0} \int_{\Omega_{R+L}} \vartheta a\left(\nabla u_{\delta}-\nabla u\right) \cdot \nabla v=0
$$

Combining (36) and (37), we infer that $\lim _{\delta \rightarrow 0} \beta_{\delta}\left(u_{\delta}-u, v\right)=0$ for all $v \in V_{R}$, which shows premise $(c)$.

We can therefore apply Theorem 2.1 and deduce the claim.

Remark 3.2 Using standard arguments for symmetric and elliptic differential operators, one can show that there exists an at most countable set $D \subset[0, \infty)$ such that for all $\omega^{2} \notin D$ the $\operatorname{kernel} \operatorname{ker}(\beta)$ is trivial.

Remark 3.3 Theorem 3.2 contains an existence result: for each $f \in L^{2}\left(\Omega_{R}\right)$ there is a unique solution $u \in V_{R}$ to (35). We mention that this existence result can also be obtained without the limiting absorption principle of Theorem 2.1. Indeed, by Lemma 3.3, the sesquilinear form $\beta=\beta_{0}$ satisfies a Gårding inequality. Thus, by Proposition 2.1 , for every $\ell \in V_{R}^{*}$ there is a unique solution $u \in V_{R}$ to $\beta(u, \cdot)=\ell(\cdot)$ provided $\operatorname{ker}(\beta)=\{0\}$.

\section{References}

1. Allaire, G., Conca, C.: Bloch wave homogenization and spectral asymptotic analysis. Journal de Mathématiques Pures et Appliquées 77(2), 153-208 (1998). DOI 10.1016/s00217824(98)80068-8 
2. Colton, D., Kress, R.: Inverse acoustic and electromagnetic scattering theory, Applied Mathematical Sciences, vol. 93, second edn. Springer-Verlag, Berlin (1998). DOI 10.1007/978-3662-03537-5. URL http://dx.doi.org/10.1007/978-3-662-03537-5

3. Conway, J.B.: A course in functional analysis, Graduate Texts in Mathematics, vol. 96, second edn. Springer-Verlag, New York (1990)

4. Dohnal, T., Schweizer, B.: A Bloch wave numerical scheme for scattering problems in periodic wave-guides. SIAM J. Numer. Anal. 56(3), 1848-1870 (2018). DOI 10.1137/17M1141643. URL https://doi.org/10.1137/17M1141643

5. Fliss, S.: A Dirichlet-to-Neumann approach for the exact computation of guided modes in photonic crystal waveguides. SIAM J. Sci. Comput. 35(2), B438-B461 (2013). DOI 10.1137/12086697X. URL http://dx.doi.org/10.1137/12086697X

6. Fliss, S., Joly, P.: Solutions of the time-harmonic wave equation in periodic waveguides: asymptotic behaviour and radiation condition. Arch. Ration. Mech. Anal. 219(1), 349-386 (2016). DOI 10.1007/s00205-015-0897-3. URL http://dx.doi.org/10.1007/s00205-015-0897-3

7. Hoang, V.: The limiting absorption principle for a periodic semi-infinite waveguide. SIAM J. Appl. Math. 71(3), 791-810 (2011). DOI 10.1137/100791798. URL http://dx.doi.org/10.1137/100791798

8. Hsiao, G.C., Wendland, W.L.: Boundary Integral Equations, Applied Mathematical Sciences, vol. 164. Springer Berlin Heidelberg (2008). DOI 10.1007/978-3-540-68545-6

9. Kreß, R.: Fredholm's alternative for compact bilinear forms in reflexive Banach spaces. J. Diff. Equations 25(2), 216-226 (1977). DOI 10.1016/0022-0396(77)90201-7. URL https://doi.org/10.1016/0022-0396(77)90201-7

10. Lamacz, A., Schweizer, B.: Outgoing wave conditions in photonic crystals and transmission properties at interfaces. ESAIM Math. Model. Numer. Anal. 52(5), 1913-1945 (2018). DOI 10.1051/m2an/2018026. URL https://doi.org/10.1051/m2an/2018026

11. Radosz, M.: New limiting absorption and limit amplitude principles for periodic operators. Z. Angew. Math. Phys. 66(2), 253-275 (2015). DOI 10.1007/s00033-014-0399-4. URL http://dx.doi.org/10.1007/s00033-014-0399-4

12. Rellich, F.: Über das asymptotische Verhalten der Lösungen von $\Delta u+\lambda u=0$ in unendlichen Gebieten. Jber. Deutsch. Math. Verein. 53, 57-65 (1943)

13. Schot, S.H.: Eighty years of Sommerfeld's radiation condition. Historia Math. 19(4), 385401 (1992). DOI 10.1016/0315-0860(92)90004-U. URL http://dx.doi.org/10.1016/03150860(92)90004-U

14. Sommerfeld, A.: Die Greensche Funktion der Schwingungsgleichung. Jahresbericht der Deutschen Mathematiker-Vereinigung 21, 309-353 (1912) 


\section{Preprints ab 2014/08}

2019-02

2019-01

2018-08

2018-07

2018-06

2018-05

2018-04

2018-03

2018-02

2018-01

2017-05

2017-04

2017-03

2017-02

2017-01

2016-05

2016-04

2016-03

\section{Ben Schweizer and Maik Urban}

On a limiting absorption principle for sesquilinear forms with an application to the Helmholtz equation in a waveguide

Michael Voit and Jeannette H.C. Woerner

Functional central limit theorems for multivariate Bessel processes in the freezing regime

Ben Schweizer

Effective Helmholtz problem in a domain with a Neumann sieve perforation

Sergio Andraus and Michael Voit

Limit theorems for multivariate Bessel processes in the freezing regime

Michael Voit

Central limit theorems for multivariate Bessel processes in the freezing regime

Elena E1 Behi-Gornostaeva, Koondanibha Mitra and Ben Schweizer

Traveling wave solutions for the Richards equation with hysteresis

Mario Ohlberger, Ben Schweizer, Maik Urban and Barbara Verfürth

Mathematical analysis of transmission properties of electromagnetic meta-materials

Margit Rösler and Michael Voit

Beta distributions and Sonine integrals for Bessel functions on symmetric cones

Michael Voit

Continuous Association Schemes and Hypergroups

Merdan Artykov and Michael Voit

Some central limit theorems for random walks associated with hypergeometric functions of type BC

\section{Ben Schweizer and Florian Theil}

Lattice dynamics on large time scales and dispersive effective equations

Frank Klinker and Christoph Reineke

A note on the regularity of matrices with uniform polynomial entries

Tomáš Dohnal and Ben Schweizer

A Bloch wave numerical scheme for scattering problems in periodic wave-guides

\section{Matthias Röger and Ben Schweizer}

Strain gradient visco-plasticity with dislocation densities contributing to the energy

Ben Schweizer and Maik Urban

Effective Maxwell's equations in general periodic microstructures

Robert Lipton and Ben Schweizer

Effective Maxwell's equations for perfectly conducting split ring resonators

Ben Schweizer

Resonance meets homogenization - Construction of meta-materials with astonishing properties

Ben Schweizer

On Friedrichs inequality, Helmholtz decomposition, vector potentials, and the div-curl lemma 
2016-01 Agnes Lamacz and Ben Schweizer

Effective acoustic properties of a meta-material consisting of small Helmholtz resonators

2015-13 Christian Eggert, Ralf Gäer, Frank Klinker

The general treatment of non-symmetric, non-balanced star circuits: On the geometrization of problems in electrical metrology

2015-12 Daniel Kobe and Jeannette H.C. Woerner

Oscillating Ornstein-Uhlenbeck processes and modelling electricity prices

2015-11 Sven Glaser

A distributional limit theorem for the realized power variation of linear fractional stable motions

2015-10 Herold Dehling, Brice Franke and Jeannette H.C. Woerner

Estimating drift parameters in a fractional Omstein Uhlenbeck process with periodic mean

2015-09 Harald Garcke, Johannes Kampmann, Andreas Rätz and Matthias Röger

A coupled surface-Cahn-Hilliard bulk-diffusion system modeling lipid raft formation in cell membrans

2015-08 Agnes Lamacz and Ben Schweizer

Outgoing wave conditions in photonic crystals and transmission properties at interfaces

2015-07 Manh Hong Duong, Agnes Lamacz, Mark A. Peletier and Upanshu Sharma

Variational approach to coarse-graining of generalized gradient flows

2015-06 Agnes Lamacz and Ben Schweizer

A negative index meta-material for Maxwell's equations

2015-05 Michael Voit

Dispersion and limit theorems for random walks associated with hypergeometric functions of type $B C$

2015-04 Andreas Rätz

Diffuse-interface approximations of osmosis free boundary problems

2015-03 Margit Rösler and Michael Voit

A multivariate version of the disk convolution

2015-02 Christina Dörlemann, Martin Heida, Ben Schweizer

Transmission conditions for the Helmholtz-equation in perforated domains

2015-01 Frank Klinker

Program of the International Conference

Geometric and Algebraic Methods in Mathematical Physics

March 16-19, 2015, Dortmund

2014-10 Frank Klinker

An explicit description of SL $(2, \mathbb{C})$ in terms of $\mathrm{SO}^{+}(3,1)$ and vice versa

2014-09 Margit Rösler and Michael Voit

Integral representation and sharp asymptotic results for some Heckman-Opdam hypergeometric functions of type BC

2014-08 Martin Heida and Ben Schweizer

Stochastic homogenization of plasticity equations 